\title{
Territorial Coordination and Workspace Awareness in Remote Tabletop Collaboration
}

\author{
Philip Tuddenham and Peter Robinson \\ University of Cambridge Computer Laboratory \\ 15 JJ Thomson Avenue, Cambridge CB3 0FD, UK \\ \{firstname\}.\{lastname\}@cl.cam.ac.uk
}

\begin{abstract}
There is growing interest in tabletop interfaces that enable remote collaboration by providing shared workspaces. This approach assumes that these remote tabletops afford the same beneficial work practices as co-located tabletop interfaces and traditional tables. This assumption has not been tested in practice. We explore two such work practices in remote tabletop collaboration: (a) coordination by territorial partitioning of space; and (b) transitioning between individual and group work within a shared task. We have evaluated co-located and remote tabletop collaboration. We found that remote collaborators did not coordinate territorially as co-located collaborators did. We found no differences between remote and co-located interfaces in their ability to afford individual and group work. However, certain interaction techniques impaired the ability to transition fluidly between these working styles. We discuss causes and the implications for the design and future study of these interfaces.
\end{abstract}

\section{Author Keywords}

Remote tabletop interfaces, territoriality, coupling, fluidity.

\section{ACM Classification Keywords}

H.5.3. Information interfaces and presentation (e.g., HCI): Group and Organization Interfaces - computer-supported cooperative work.

\section{INTRODUCTION}

In recent years, tabletop interfaces have emerged as an effective tool to support co-located collaboration around a shared digital workspace for a wide variety of tasks. They afford some of the familiar work practices of collaboration around traditional tables, such as fluid transitioning between individual and group work [15, 19, 20], and coordination based on spatial partitioning $[16,17]$.

Permission to make digital or hard copies of all or part of this work for personal or classroom use is granted without fee provided that copies are not made or distributed for profit or commercial advantage and that copies bear this notice and the full citation on the first page. To copy otherwise, or republish, to post on servers or to redistribute to lists, requires prior specific permission and/or a fee.

CHI 2009, April 4-9, 2009, Boston, Massachusetts, USA.

Copyright 2009 ACM 978-1-60558-246-7/09/04 ..\$5.00.
Lately there has been growing interest in systems connecting two geographically-separated tabletop interfaces together to support remote collaboration $[1,8,9,14,18$, 23]. These remote tabletop interfaces typically provide a large horizontal shared workspace in which remote collaborators see each other's interactions with virtual task artefacts, such as digital photos, documents, or sketches, along with remote "shadow" representations of the arms at the remote site. Like tabletop interfaces, they typically enable several collaborators to work concurrently to move and otherwise manipulate virtual task artefacts using direct input devices such as touch surfaces or styluses. Figure 1 illustrates such a system.

Underlying the design of remote tabletops is an assumption: that mimicking tabletop interfaces in this way will afford for remote collaborators the same beneficial work practices as co-located tabletop collaboration, such as fluid transitioning between individual and group work, and coordination based on spatial partitioning. If true, this may provide a more effective remote collaboration medium than conventional groupware, which has limited support for the work practices and visual cues of co-located collaboration
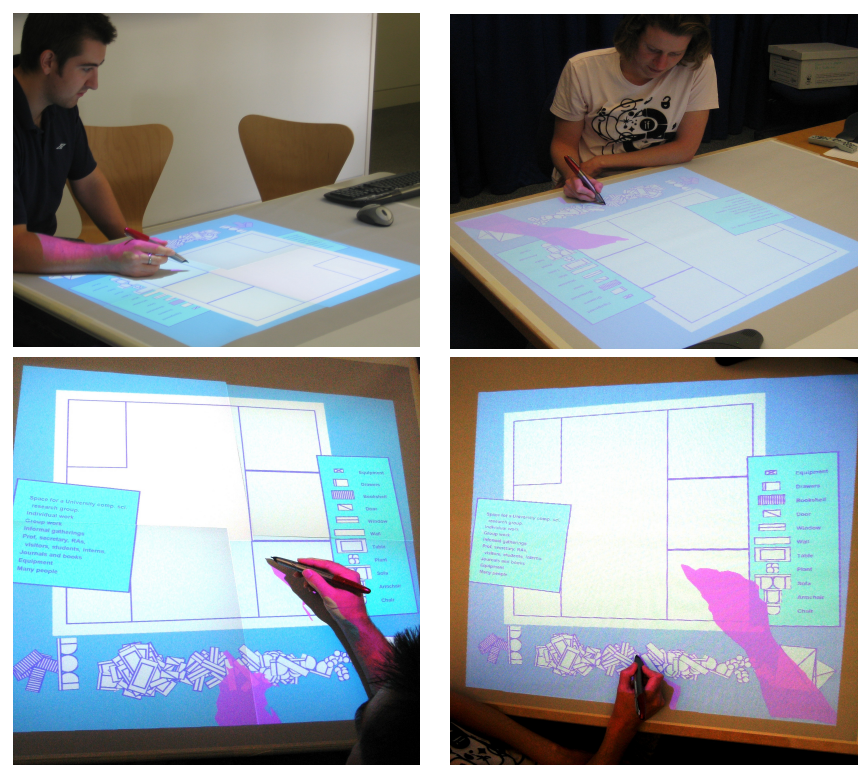

Figure 1. Two tabletop interfaces are linked to provide a large shared workspace with purple remote arm "shadows". 
[5]. However, the assumption has not been tested in practice. Previous studies of remote tabletops have focused on the remote arm representations, showing that they support a variety of workspace-oriented gestures [10] and aid remote collaborators in maintaining awareness of each other's actions [18]. Beyond this, there has been little investigation and so we do not know whether the assumption holds. In contrast to the wealth of knowledge about co-located tabletop collaboration, we know little about the work practices afforded by remote tabletops, and the effects of interaction design.

Findings from other, more widely-studied, synchronous remote collaboration interfaces are difficult to apply directly to this problem. Studies of remote whiteboard interfaces [e.g. 21] have been limited to spatially-fixed sketching rather than the design and layout tasks or tabletop interaction techniques of remote tabletop interfaces. Remote physical systems provide remote collaborators with video views of physical task artefacts [e.g. 10, 24]. Typically only a single collaborator can interact with the task artefacts during a session and so, while these systems lend themselves to instructor-follower assembly tasks, the work practices are likely different in remote tabletop interfaces in which both collaborators can interact. Lastly, conventional groupware applications create a shared workspace using a monitor, mouse, and GUI approach [e.g. 3, 4, 5]. They differ from remote tabletops in form factor, in interaction design, and in remote gesture representation.

In this paper, we present an exploratory study comparing remote and co-located tabletop collaboration. We focus on two areas. Firstly we examine coordination using territorial partitioning of space, and observe key differences in this work practice between remote and co-located tabletop collaboration. Secondly, we examine workspace awareness which, as we explain, is necessary for the fluid transitioning between individual and group work observed in co-located tabletop collaboration. We find no differences between study conditions but observe that some interaction techniques enabled fluid transitioning while others impaired this practice. The findings inform the design of remote and co-located tabletop interfaces, and groupware at large, and also provide insights into tabletop work practices.

\section{BACKGROUND}

\section{Territorial Coordination}

When collaborators sit around a traditional table, each has a distinct area of table in front of them in which they can carry out individual work as part of the task, for example to try out ideas away from the group. Tang [20] observed a colocated group design task using pieces of paper on a table, and noted that sketches drawn in this area are "within a personal boundary and not intended for others to perceive". Scott et al. [17] observed a tabletop design task, again using paper, and found that collaborators can move task artefacts, such as pieces of paper and tools, into this personal territory to reserve them for themselves, and can later move them back towards the centre of the table to indicate their availability to the group. Collaborators also can implicitly partition space in the centre of the table so that each takes responsibility for the nearest region. Scott et al. [16] later observed similar territorial behaviour in a tabletop interface using a collaborative photo-layout task. Collaborators would, for example, position a virtual container of digital photos centrally on the table when working together, and when working individually would move containers into and out of their work area as necessary without disrupting their partner. Territoriality is therefore a coordination mechanism key to tabletop collaboration. We do not, however, know whether remote tabletop interfaces will afford this practice.

Remote tabletop collaborators can either arrange themselves around the table, as they would when colocated, or alternatively can both sit in the same position relative to the workspace. Collaborators in this second, overlaid, arrangement are unlikely to be territorial since their personal areas overlap, and so may have difficultly coordinating their work. Seating preferences in remote tabletop collaboration are unclear. Kirk [10] studied a remote instructor-follower physical-assembly system and found a non-significant trend that an overlaid arrangement was easier for participants and resulted in more progress than non-overlaid arrangements. However, the instructorfollower nature of the task and system meant that territorial coordination was neither possible nor advantageous. By contrast, co-located collaborators prefer to interact at a distance that preserves their personal space [7].

\section{Workspace Awareness}

Gutwin and Greenberg define workspace awareness as "the up-to-the-moment understanding of another person's interaction with the shared workspace" [5]. They show that workspace awareness is necessary for collaborative coupling, the way in which collaborators transition regularly between working individually and working closely with others, even in a shared task. Such transitions tend to be opportunistic and unpredictable [3]. Collaborators may transition from individual work to closely working with others because, for instance, they need to discuss a decision with each other, or because one has reached a point in their individual work that needs another's involvement. Gutwin and Greenberg [5] argue that supporting this work practice is important, but difficult. Each collaborator must be aware of the state of their peers and of the task in order to spot appropriate opportunities to instigate discussion or individual work, and to recognise when others are trying to do so. Coupling therefore relies on workspace awareness. Workspace awareness also underpins other work practices, such as coordinating intricate actions when working closely together; anticipating the actions of others; and offering assistance.

Gutwin and Greenberg [5] also show how collaborators maintain awareness not only by conversing and gesturing to each other, but also by watching each other work, either 


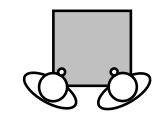

Co-locatedadjacent (CA)

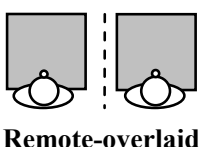

(RO)

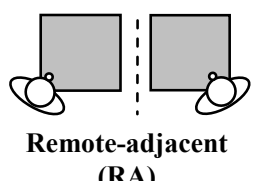

(RA)
Figure 2. Study conditions.

directly or peripherally. They maintain awareness by consequential communication (watching a collaborator's arms), and by feedthrough (watching changes in task artefacts as they are manipulated by a collaborator's arms). Such visual cues are often impoverished in remote collaboration systems, and so an explicit consideration of awareness is important when investigating remote tabletop collaboration.

Tang [20] observed a group design task at a traditional table and noted how a peripheral awareness of each other's activities enables collaborators to coordinate intricate actions and transition fluidly between working individually and working closely with others. A number of studies of tabletop interfaces have reported similar observations in tasks such as information-gathering and planning [15], photo-layout [16], and route-planning [19], suggesting that tabletop interfaces too can afford high levels of workspace awareness and this work practice. Tse et al. [22] consider workspace awareness when designing a speech and gesture interface for co-located tabletop collaboration.

Tang et al. [18] consider workspace awareness when designing remote arm shadow representations for remote tabletop collaboration. In a preliminary study, they observed that the remote arms help collaborators to watch each other work, suggesting they enable consequential communication. However, arm representation is only one aspect of design for awareness. In conventional groupware, for instance, interaction techniques are also important, such as the way in which collaborators manipulate task artefacts [4]. We know little of how interaction techniques in remote collaboration affect workspace awareness, so it is unclear how to design remote tabletops to provide high awareness and support the desirable work practices that require it.

\section{EXPLORATORY STUDY}

This study accordingly investigates two questions:

- Do remote tabletops afford territoriality as a coordination mechanism, and how is this affected by seating arrangement?

- How does the design of remote tabletops impact workspace awareness and the practices that depend on it?

\section{Technology}

We used the Distributed Tabletops system [23] (Figure 1). It provides a large shared workspace in which co-located or remote collaborators can interact concurrently using digital styluses to move, reorient, and otherwise manipulate task artefacts. Remote collaborators' arms are shown as translucent "shadows". Each collaborator's shadows are also displayed locally to provide feedback of the remote representation. The system runs at $60 \mathrm{fps}$ with latency around $100 \mathrm{~ms}$. Arm shadows are captured at $15 \mathrm{fps}$. Sites were also linked using a speakerphone.

\section{Study Design}

The study investigated three conditions (Figure 2):

- Co-located-adjacent (CA): Collaborators sat at the same table, positioned at adjacent corners. Collaborators had their own styluses and could interact concurrently.

- Remote-adjacent (RA): Collaborators sat in separate rooms at different tables, which were linked using the system. Collaborators were again positioned at adjacent corners. The rooms were linked using a speakerphone. Again, both collaborators could interact concurrently.

- Remote-overlaid (RO). As remote-adjacent, except that collaborators sat in the overlaid seating arrangement.

We used a within-subjects design, so each pair of subjects tested each of the three conditions. The presentation order of the conditions was counterbalanced across the pairs using a Latin square. Pairs used the system to fulfil each of three design briefs in turn, one in each condition. The presentation order of the briefs was counterbalanced appropriately across the conditions and the pairs. Data was analysed from system $\log$ files and video recordings. Participants were asked about preferences and difficulties in post-condition and post-study questionnaires, and semistructured interviews.

\section{Task and Interaction}

Participants were asked to work together to arrange diagrammatic furniture on a floor plan to fulfil a design brief. For example, one such brief asked participants to design a communal space for graduate students and to provide, among other things, as much seating as possible, and areas for serving drinks. The other briefs were similar and asked for designs for a library and for a research lab. Participants were asked to colour the furniture items to show how they had fulfilled different parts of the brief. At the end of the task, participants gave a short joint presentation and answered questions about their solution.

This task is representative of tabletop design tasks. It entails discussion and exploration of different approaches and tradeoffs by manipulating task artefacts. Participants were asked to use their prior experiences of communal spaces, and so many constraints were implicit. This, with the tradeoffs and joint presentation, helped avoid a purely divide-and-conquer approach. Instead, participants had to remain aware of each other's actions and coordinate if they were to produce a satisfactory outcome.

The shared workspace was a $75 \mathrm{~cm} \times 75 \mathrm{~cm}$ blue square and started with an empty white floor plan, "piles" of diagrammatic furniture, the task brief, and a key explaining the furniture representations (Figure 1). The floor plan was empty except for lines marking room boundaries. 


\begin{tabular}{|l|c|c|c|}
\hline Question & CA & RO & RA \\
\hline $\begin{array}{l}\text { "We worked together throughout the task." } \\
\text { (1=strongly agree, 7=strongly disagree) }\end{array}$ & $\begin{array}{c}2.0 \\
(0.8)\end{array}$ & $\begin{array}{c}2.1 \\
(1.2)\end{array}$ & $\begin{array}{c}2.1 \\
(0.9)\end{array}$ \\
\hline $\begin{array}{l}\text { "How easy or hard was the task to complete using } \\
\text { this technology?" (1=very easy, 7=very hard) }\end{array}$ & $\begin{array}{c}2.5 \\
(0.9)\end{array}$ & $\begin{array}{c}2.2 \\
(0.9)\end{array}$ & $\begin{array}{c}2.4 \\
(0.8)\end{array}$ \\
\hline $\begin{array}{l}\text { "How did you find communicating this way?" } \\
\text { (1=very easy, 7=very hard) }\end{array}$ & $\begin{array}{c}1.7 \\
(0.8)\end{array}$ & $\begin{array}{c}1.9 \\
(0.6)\end{array}$ & $\begin{array}{c}2.3 \\
(0.8)\end{array}$ \\
\hline
\end{tabular}

Table 1. Mean (standard deviation) Likert scale responses.

All task artefacts (including the plan) could be freely moved using a stylus. We used the popular Rotate ' $\mathrm{N}$ ' Translate interaction technique [11] in which task artefacts can be simultaneously rotated and translated with a single stylus stroke by using a pseudo-physics model. Both collaborators could work concurrently to move different task artefacts. If the floor plan was moved, any furniture on it would move with it. Furniture on the floor plan snapped its orientation to multiples of $45^{\circ}$. Tapping twice on a furniture item opened a menu on which the participant could tap to change the item's colour.

\section{Participants and Procedure}

18 paid participants (aged 20-39, 16 males) from a Computer Science department formed 9 pairs $(2$ mixed-sex pairs). Partners in each pair had met previously. Two were left-handed. One had limited experience with tabletop interfaces; the others had none.

After a short tutorial, participants were arranged in the first condition. For each condition, participants were arranged appropriately and asked to stay in that arrangement. Participants then practised together until comfortable using a practice brief. Pairs took about ten minutes to practise before their first condition, and less than two minutes thereafter. The task brief was then loaded and read, and the pair was asked to fulfil the brief to the best of their abilities. At the end of each condition, each pair gave a short presentation, answered questions about their design, and completed individual post-condition questionnaires. When all three conditions were finished, they completed a poststudy questionnaire and a semi-structured interview.

\section{RESULTS: INITIAL OBSERVATIONS}

Pairs worked for an average of 21 minutes in each condition. In all conditions they engaged in meaningful design collaboration, using their prior knowledge and exploring different layouts to produce appropriate outcomes. They engaged in both individual and group work, both taking turns and working concurrently.

Participants used the arm representations to convey a variety of workspace gestures: deictic gestures, by pointing at a room or waving over an area; spatial gestures, using the hand to indicate shapes and trace paths; and action gestures to indicate, for example, turning. They seemed to have no difficulty interpreting these gestures.

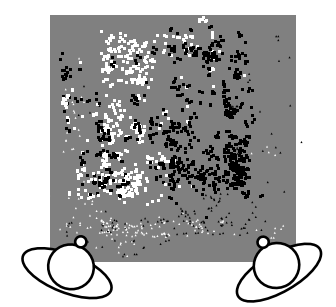

(a) Co-locatedadjacent (CA)

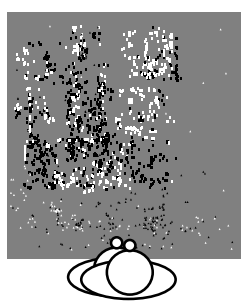

(b) Remoteoverlaid (RO)

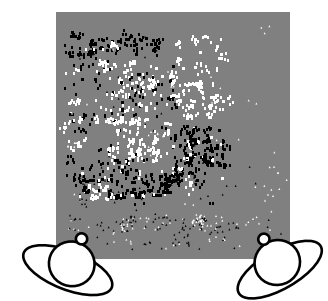
adjacent (RA) (c) Remote-

Figure 3. Activity map showing interactions of one pair in the workspace. Each marker corresponds to a task artefact being picked up or dropped. Colour indicates the person interacting.
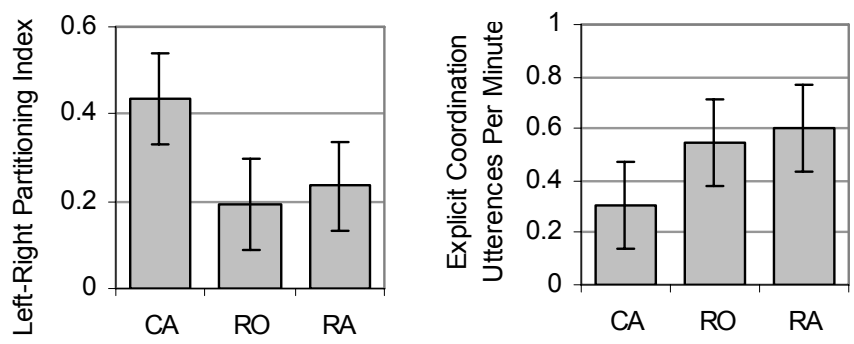

Figure 4. Left-right partitioning index and rate of coordination utterances for each condition. Error bars are $\mathbf{9 5 \%}$ confidence intervals and indicate variation within pairs (i.e. the error considered by a repeated-measures ANOVA).

Pairs in all three conditions seemed to have few difficulties interacting with the system and each other. Post-condition questionnaires asked about ease of task completion, ease of communication, and the extent to which the pair worked together, using 7-point Likert scales (Table 1). Friedman rank tests for repeated-measures data yielded no significant differences among conditions. When asked their preference for remote seating arrangement, 11 preferred overlaid, 6 preferred adjacent, and 1 didn't know. A chi-squared test found no significant preference.

\section{RESULTS: TERRITORIAL COORDINATION}

Figure 3 shows activity maps [17], generated from log files, which illustrate the location of a single pair's interactions with furniture in each condition. The distribution of black and white in Figure 3a suggests that when co-locatedadjacent they tended to partition the space so that each participant worked on the side of the table nearest to themselves. By contrast, the distribution of black and white in Figures $3 b$ and $3 c$ suggests that partitioning in the remote conditions formed a patchwork rather than a strict left-right arrangement.

To further investigate, we quantify the extent to which participants' interactions were partitioned to the left and right. We follow the approach of Scott et al. [17] in examining the proportion of interactions carried out by each participant on each side of the table. The left-hand side might, for example, have been $30 \%$ participant $\mathrm{A}$ and $70 \%$ participant B. However, this tells us little about partitioning because $\mathrm{B}$ may have been more active than $\mathrm{A}$ on the right- 


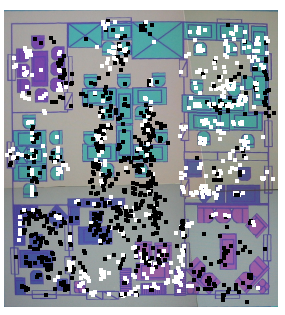

Remote-overlaid

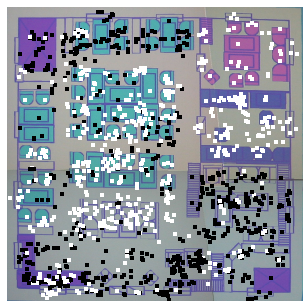

Remote-adjacent
Figure 5. Interactions of one pair overlaid on their final furniture layouts. The "furniture" was coloured (blue, purple, etc.) by participants during the task to show how they had fulfilled different parts of the brief. "Walls" are shown as blue lines. Each marker corresponds to a task artefact being picked up or dropped. Marker colour (black or white) indicates the person interacting.

hand side also. Similarly, calculating that, for instance, $80 \%$ of B's interactions were on the right-hand side of the table tells us little about partitioning since $80 \%$ of A's interactions may also have been on the right-hand side.

Instead, we calculate the degree of partitioning between collaborators. For partners $\mathrm{A}$ and $\mathrm{B}$, the left-right partitioning index is computed by first calculating the proportions of A's interactions that lie within the left-hand side of the table, and then the same for B's, and then taking the absolute difference between these. If, say, $70 \%$ of A's interactions were on the left, and $30 \%$ of B's, this yields an index of $|0.7-0.3|=0.4$. If all of A's interactions were on the left, and none of B's were on the left, this yields an index of $|1.0-0.0|=1.0$. If both participants interacted equally on the left, this yields an index of 0 . This aggregate measure serves to highlight quantitatively differences in partitioning.

Figure 4 shows the index for each condition. The results are consistent with the trends identified in the activity maps. In the co-located-adjacent condition, the mean index of 0.4 corresponds to the $70 \%: 30 \%$ split above. In both remote cases, the mean index of 0.2 corresponds to a $60 \%: 40 \%$ split. Curiously, the degree of left-right partitioning in the remote-adjacent condition was indistinguishable from that of the remote-overlaid condition, in which participants sat in the same position with respect to the workspace and so were not able to partition by proximity at all.

This difference was confirmed by a one-way repeatedmeasures ANOVA $(\mathrm{F}(2,16)=7.02, p<0.01)$. Pairwise $\mathrm{t}$-tests found significant differences between $\mathrm{CA}$ and $\mathrm{RA}$ $(\mathrm{t}(8)=3.64, p<0.01)$, and between CA and RO $(\mathrm{t}(8)=2.83$, $p=0.022$ ).

In order to further investigate the patchwork partitioning of the remote conditions, we transformed and overlaid the marker locations on the participants' final furniture layout (Figure 5). The resulting patchworks of black and white markers show how participants spatially partitioned the task. This patchwork partitioning seemed to follow either the "walls" on the floor plan at the outset of the task, or

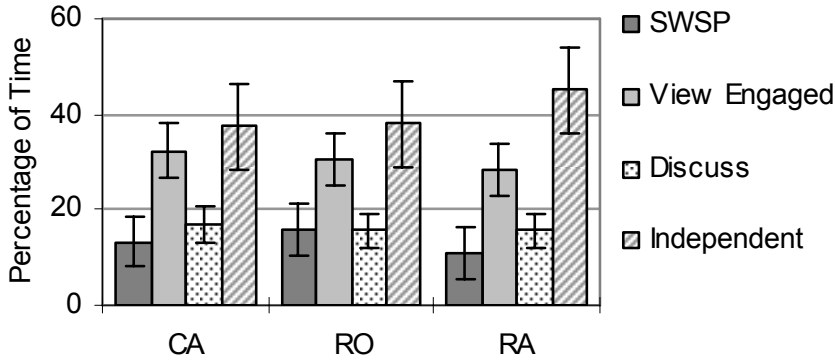

Figure 6. The proportion of time spent in each coupling style in each condition. Error bars indicate variation within pairs and show $95 \%$ confidence intervals.

islands of "furniture" or new "walls" created by the participants during the task.

Participants in the remote conditions did not follow social norms and partition based on proximity as when co-located, suggesting they may have done extra work to coordinate their activities. Though much of the coordination seemed implicit in their actions, they also used explicit coordination utterances to communicate to each other what they had done or should do (e.g. "I'll do the common room now", "I've finished doing the windows", "You can start on the secretary's room"). The dialogue was coded for such utterances. Figure 4 (right) shows that such explicit coordination utterances were on average twice as frequent in the remote conditions. This was confirmed by a one-way repeated-measures ANOVA using the conservative Greenhouse-Geisser sphericity correction $(\mathrm{F}(2,16)=6.142$, $p=0.03$ ). Pairwise $\mathrm{t}$-tests found significant differences between CA and RO $(\mathrm{t}(8)=4.25, p<0.01)$, and between CA and $\mathrm{RA}(\mathrm{t}(8)=2.40, p=0.04)$.

\section{RESULTS: WORKSPACE AWARENESS}

We now consider how the interaction techniques affected workspace awareness. We focus on consequential communication and feedthrough, since other research has investigated intentional gesture in detail [e.g. 10]. However, consequential communication and feedthrough are not easily observed directly. Instead, we begin by analysing their effects on collaborative coupling.

\section{Coupling Styles}

We iteratively refined a coding scheme for coupling styles, repeatedly analysing selected segments of video, using a similar approach to prior studies of co-located collaboration $[17,19,20]$. Initial coding categories were informed by tabletop coupling styles identified previously by Tang et al. [19]. This yielded four styles that classify the coupling arrangement at any time:

- Simultaneous work on the same problem, (SWSP): The collaborators are actively working together simultaneously to help each other solve the same problem, such as both arranging the windows.

- View engaged: As Tang et al. [19]. The collaborators are working together but one is manipulating the 
display while the other watches closely, such as demonstrating ideas to each other or taking turns.

- Discuss: The collaborators are working together but conversing rather than manipulating the display.

- Independent work: Collaborators are working independently, sometimes interacting and sometimes looking at the workspace. Collaborators often glanced at each other's work areas. Conversation varied from silence to rapid chit-chat.

We analysed the entire video of each session to determine the proportion of time spent in each coupling style and thus whether different conditions affected ability to collaborate in different styles. As might be expected with a quasinaturalistic task, there was a large variation between pairs in the proportion of time spent in the coupling styles. Some pairs tended to stay closely-coupled throughout, whereas others worked mostly independently. Nevertheless, within each pair, we did not observe large differences among conditions in the proportion of time spent in each style (Figure 6). Repeated-measures ANOVAs found no significant differences.

Surprisingly then, we did not observe that moving from colocated to remote had any practical impact on participants' behaviour at this aggregate level, for this fairly representative design task. They seemed able to work both independently and closely coupled to the same extent regardless of the condition.

\section{Coupling Transitions}

During the video analysis, we observed that participants seemed able in all conditions to transition fluidly among coupling styles. Transitions seemed rapid and opportunistic, instigated not by explicit gestures or conversation, but instead by watching each other's arm movements in the workspace (consequential communication) and each other's manipulations of task artefacts (feedthrough).

Consider the representative example in Figure 7. Collaborators $\mathrm{L}$ and $\mathrm{R}$, are working independently in different areas of the workspace (Figure 7a). L glances and observes $\mathrm{R}$ arranging furniture in a corner, and begins to watch (view engaged, Figure $7 b$ ). L then assists $R$ by moving a door, positioning his arm to avoid obscuring R's activity (simultaneous work on the same problem, Figure 7c). Only at this point does either speak. Collaborators also transitioned from independent working to anticipate and assist in other ways, such as by watching and then providing verbal suggestions. This typically led into a session of turn-taking in which collaborators watched each other demonstrate different ideas.

Although these fluid coupling transitions are desirable, and indicate high awareness levels, it was not clear how they arose from the interaction design. However, we also observed that two interaction techniques used occasionally by participants tended to result in one participant's actions
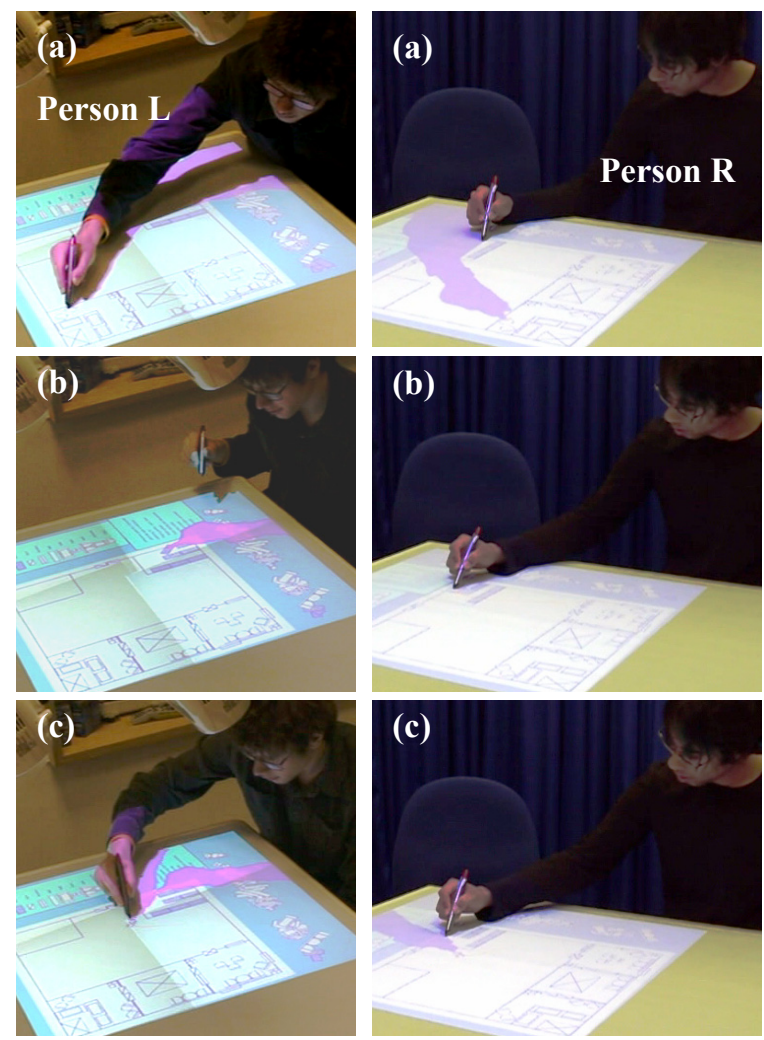

( $L$ and R work independently. L glances at R's work.) (a)

( $L$ stops work and watches R.) (view engaged) (b)

$L$ : eh it's good need a door

( $L$ assists $R$ by adding a door to the room that $R$ is arranging.) (simultaneous work on the same problem) (c)

Figure 7. A series of coupling transitions.

being unanticipated by their partner, and consequently led to confusion that was resolved verbally. Consideration of how these techniques impaired workspace awareness provides insights as to how the remainder of the system afforded the otherwise-high awareness level.

The first occasional problem occurred when participants moved the large floor plan. Using the popular Rotate ' $\mathrm{N}$ ' Translate technique [11], the plan could be rotated and translated by touching any part of it with the stylus and then dragging. This action often seemed unanticipated by the instigator's partner. Figure 8 shows a representative example. L says he is going to spin the floor plan, and $\mathrm{R}$ starts to reach with his hand to the top of the plan, presumably to interact (Figure 8a). Just as his hand reaches the top of the plan, however, L spins the plan (Figure 8b). R has to abort the reaching action, retracting the hand (Figure $8 c$ ) and saying "oh I see yeah".

This contrasts with the fluid coupling transitions when moving furniture (Figure 7), and yet both cases used Rotate ' $N$ ' Translate. The difference seems then due to the large size of the floor plan. Furniture items are small and so a collaborator must reach towards an item to move it. This reaching action can be peripherally observed by their 

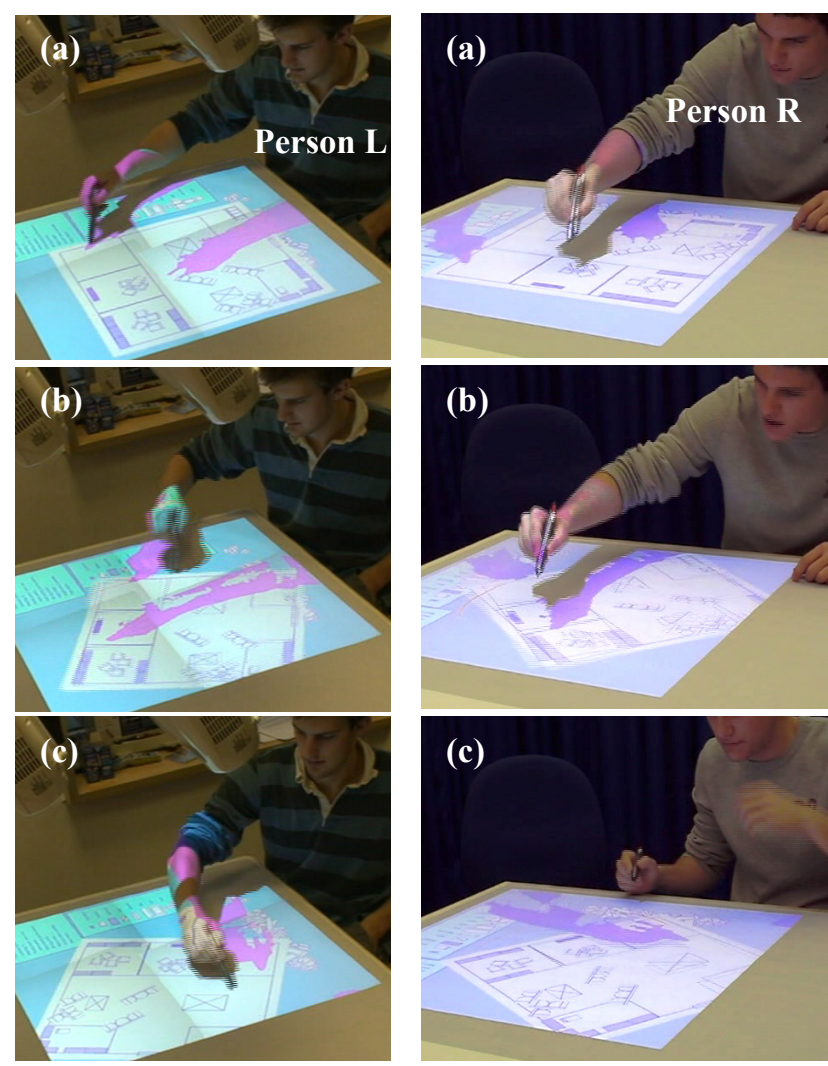

L: do you want to spin the plan so you can get at it more easily

( $R$ reaches towards the top of the plan start working) (a)

( $L$ starts to rotate the plan. $R$ has not anticipated this and has to abort the reaching action.) (b)

( $L$ still rotates the plan. $R$ backs off looking puzzled.) (c)

$\mathrm{R}$ : oh I see yeah

Figure 8. Deliberate movement of the floor plan by one participant was unanticipated by their collaborator.

partner (consequential communication). By contrast, the floor plan can be moved by dragging from anywhere within its large area, without requiring reaching to a specific location, and so was harder to anticipate. In Figure 8a, for instance, it is not clear from R's view that $\mathrm{L}$ is about to rotate the plan. Furthermore, furniture items are small so their movement is localised near to the interacting hand shadow. A collaborator might then assume that if their partner's hand shadow is far from their own then their actions will not interfere. This is not true when moving the floor plan, which is so large that its motion is not localised to the vicinity of the interacting hand.

A second occasional problem occurred when participants tapped twice on a furniture item to open the colour menu. Again, this action often seemed unanticipated by the instigator's partner. Figure 9 shows a representative example. L has opened a colour menu (Figure 9a). As she presses on it, R opens another colour menu that obscures it. L unintentionally presses on this second menu instead, causing the wrong item of furniture to change to the wrong colour. Both collaborators are confused and begin
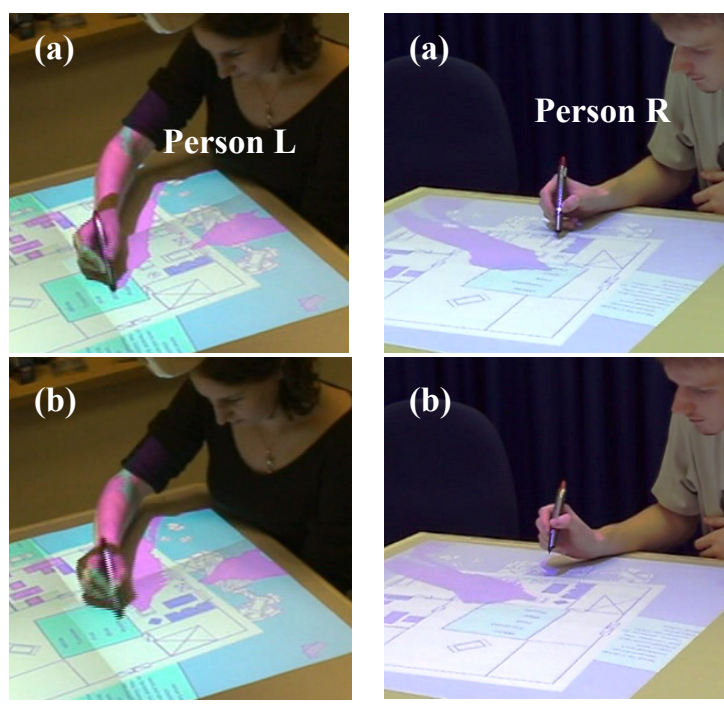

( $L$ has opened a menu and is about to press on it.) (a)

( $R$ opens a menu as $L$ presses, causing $L$ to press on this new menu instead of her own menu. Consequently, the wrong item of furniture changes colour.) (b)

L: oh?

Figure 9. Opening of a menu by one participant was unanticipated by their partner.

discussion (Figure 9b). This again contrasts with the fluid coupling transitions when moving furniture (Figure 7).

In Figure 9a, the tapping action by $\mathrm{R}$ is not apparent from the arm shadow seen by $\mathrm{L}$, and so provides no consequential communication. Furthermore, the menu appears instantaneously and only once the tapping action is complete, providing no feedthrough as the action unfolds. By contrast, the furniture in Figure 7 moves with the shadow of the dragging arm (consequential communication). The movement of the furniture itself is continuous and incremental, not instantaneous, and so is perceptible as the action unfolds (feedthrough).

\section{DISCUSSION}

\section{Territorial Coordination}

We found quantitatively that the study condition affected collaborators' partitioning of the workspace. Co-locatedadjacent collaborators partitioned the space according to who was nearest. This agrees with Scott et al. [17], who found that collaborators at traditional tables implicitly assume responsibility for the part of the group space closest to themselves. Their study involved two co-located pairs, and we calculate the left-right partitioning indices from their data as 0.51 and 0.42 , which agree with our results.

Remote-overlaid collaborators did not have the opportunity to partition and delegate on the basis of proximity. Instead, each pair partitioned the floor plan into a patchwork, using existing visible boundaries or creating new ones.

It is perhaps surprising that, despite having the adjacent seating arrangement, remote-adjacent collaborators 
condition also did not use the proximity-based partitioning observed in the co-located-adjacent condition. This suggests that territorial coordination arises from more than mere seating arrangement.

Observations of reaching may go some way to explaining the differences between co-located-adjacent and remoteadjacent. Co-located-adjacent collaborators seemed wary of reaching across their partners for fear of blocking them from working. Before reaching across to access the floor plan or furniture, they would typically wait for an opportune moment, and sometimes ask permission (e.g. Figure 10). In both remote conditions, however, participants worked across each other, worked in shadows, and took furniture from in front of each other all without hesitation or utterance (e.g. Figure 7). This blocking hypothesis is supported by other recent findings. Co-located tabletop collaborators use less proximity-based coordination when using action-at-a-distance techniques like "tractor beams" (which avoid blocking problems) than when using conventional techniques like Rotate'N'Translate [13]. In another study, a majority of participants reported being more likely to interact with objects on their partner's side of the table when using a mouse (which avoids blocking problems) rather than a stylus [6].

The differences in spatial partitioning and observations of blocking suggest that remote tabletops do not support the work practice of territoriality observed in co-located collaboration at traditional tables and tabletop interfaces. As described earlier, the tabletop region immediately in front of each collaborator serves as a personal territory in which to try out ideas away from the group, and to place task artefacts to reserve them for their own use [17]. This will not be the case in the remote-overlaid condition, in which these regions are overlaid. That remote-adjacent collaborators took items of furniture from in front of each other without hesitation or utterance suggests that they also do not respect personal territories.

There are two supplementary results. Firstly, like Kirk [10], we found a non-significant trend for remote collaborators preferring the overlaid arrangement. 10 of the 11 who preferred this said in questionnaires that they had difficulty reaching some table areas when remote-adjacent. Curiously, none mentioned this when co-located-adjacent, though they were sat in the same positions. Observations suggest this difference may be due to the remote gesture system. Colocated-adjacent participants could point to far-away parts of the table by using the 3-D trajectory of their finger. Remote collaborators were instead observed reaching out to hover their hands over far-away parts of the table in order to point, presumably because the remote gesture representation did not convey $3-\mathrm{D}$ depth cues. This reaching seemed more awkward in the remote-adjacent condition, where collaborators were positioned to one side. This may have contributed to the preference. Secondly, the patchwork partitioning coincided with a greater rate of explicit verbal coordination. This suggests that patchwork partitioning,
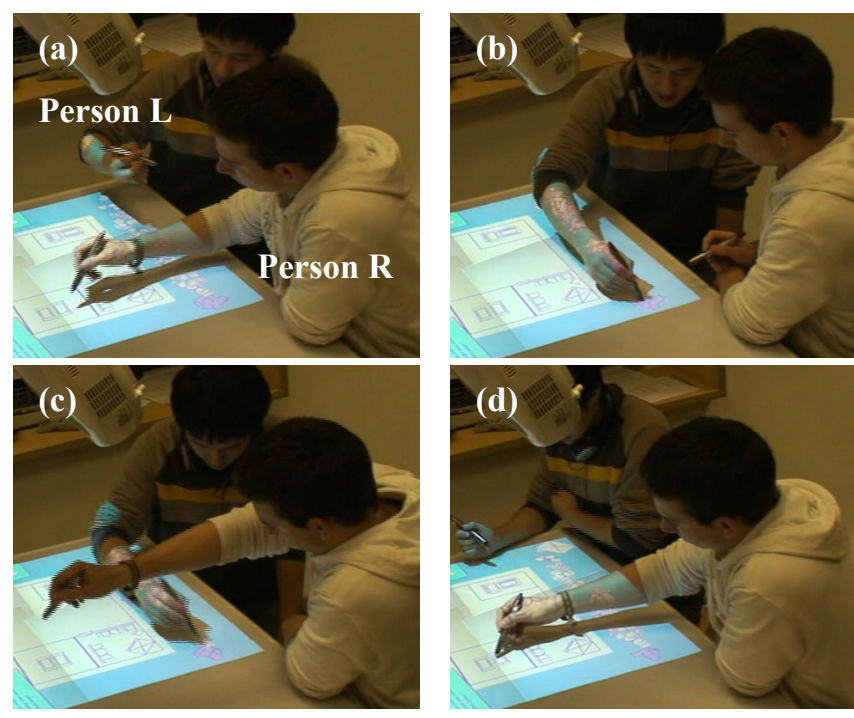

( $R$ and $L$ are working independently.) (a)

$\mathrm{L}$ : yeah I'll start doing pigeon holes

R: okay

( $R$ leans back and $L$ leans across and takes a bookshelf.) (b)

$\mathrm{L}$ : um bookshelves are pigeon holes can I just steal like loads?

( $L$ leans across to take more and $R$ tries to work.) (c)

( $L$ finishes taking and $R$ leans forward to work again.) (d)

$\mathrm{L}$ : cheers

Figure 10. Co-located-adjacent collaborators have difficulty working across each other.

while less constrained than territorial partitioning, may lead to greater coordination effort to decide who does what.

\section{Workspace Awareness}

We examined collaborative coupling, a beneficial work practice that both requires a high awareness level and has been observed in co-located tabletop collaboration. We found no significant differences among conditions in the proportion of time collaborators spent in each of four identified coupling styles. The confidence intervals on the proportions suggest that moving between conditions had relatively little practical impact on collaborators' inclinations to work in different styles. (By contrast, Tang et al. [19] found that manipulating a tabletop interface design led to a fifty percentage-point difference in the proportion of time spent in similar coupling styles.) This is not to claim that the conditions were identical; a difference might be more pronounced given an alternative task. Nevertheless, for this fairly representative design task, in situations that the collaborators themselves constructed as they proceeded through the task, the different conditions had relatively little practical impact at this aggregate level.

We observed that interaction techniques seemed to affect collaborators' abilities to transition fluidly between coupling styles. Two techniques used occasionally by the collaborators tended to impair workspace awareness and resulted in confusion that was resolved verbally. Firstly, movement of the floor plan could be instigated from any 
point and so collaborators did not have to reach to instigate the action. This lack of consequential communication made the action hard to anticipate. Secondly, the double-tap action to open the menu was not conveyed by the arm shadow (no consequential communication) and the menu appeared only instantaneously at the end of the action (no feedthrough), and so was also hard to anticipate.

By contrast, most transitions seemed fluid and opportunistic, relying on workspace awareness maintained through consequential communication and feedthrough as collaborators moved furniture items. Collaborators also offered assistance and closely-coordinated their actions when working in the same room; further indication of a high awareness level. Movement of a furniture item was localised to the vicinity of the interacting hand and collaborators had to reach towards the item to instigate movement (consequential communication). The movement of the item itself presented continuous incremental visual changes that could be observed by collaborators as the action unfolded (feedthrough).

\section{Implication 1: Visual boundaries to aid coordination}

Co-located tabletop collaborators partition the space based on proximity, whereas remote collaborators use a patchwork partitioning based on visual boundaries. In the overlaid arrangement there is no personal territory in which to reserve task artefacts and carry out individual work, and our observations suggest that remote-adjacent collaborators do not respect personal territory either. The use in a remote setting of applications and techniques that have been designed for co-located tabletop interaction is therefore likely to lead to coordination difficulties. Providing flexible visual boundaries for remote tabletop collaborators may alleviate this problem. For example, providing each collaborator with a moveable coloured palette region onto which task artefacts could be placed may serve as a personal territory. When predetermined boundaries are not appropriate, designers could instead enable collaborators to sketch and amend their own visible boundaries.

\section{Implication 2: Localised and continuous incremental visual changes}

Our observations suggest that high awareness level and fluid transitions between coupling styles can arise from interaction techniques that (a) localise visual changes to the vicinity of the interacting hand, and (b) localise the area in which the action can be instigated, and (c) produce continuous incremental visual changes. Two techniques that did not follow these properties resulted in actions that proved difficult to anticipate, and led to confusion.

These properties of localised and continuous incremental visual changes are fulfilled by tabletop interfaces in which collaborators use techniques like Rotate'N'Translate [11] to move small task artefacts (such as furniture items in this study, or photos [e.g. 16]). The resulting high awareness level is perhaps one reason why these interfaces successfully support collaborative activity. The properties are also fulfilled by a number of other tabletop interaction techniques, such as dragging from a pile to create new task artefacts, dragging to a "recycle bin" to remove task artefacts, and using individual movable lenses [19] to switch visualisation mode.

We can also use these properties to consider alternative techniques for opening menus and dragging large task artefacts that, our experiences suggest, will not impair awareness. A menu could appear gradually as the collaborator drags from a point, so that both hand action and menu opening are observable as the action unfolds. Alternatively the menu may be kept permanently open as a box [e.g. 12] or toolglass [2] so it becomes a small movable task artefact whose interactions, like the small furniture items, yield high awareness. The visual effects of dragging a large task artefact cannot be localised to the dragging hand because of its size. We can instead localise the area in which the action can be instigated to a small "drag handle" attached to the task artefact so that collaborators can see each other reaching towards the handle and so anticipate the action.

The proposal for continuous incremental visual changes to promote awareness in tabletop collaboration is similar to the argument of Gutwin and Greenberg [4] that conventional GUI groupware should follow such principles. However, we believe that the use of localised interaction to potentially boost awareness has not previously been investigated. Furthermore, just as continuous incremental visual changes increase awareness at the expense of reduced individual power (e.g. by prohibiting fast keyboard shortcuts) [4], so a similar trade-off applies to locality: encouraging reaching by localising the point of instigation may boost awareness but requires extra reaching effort by individuals. This may ultimately result in unacceptable fatigue. Nevertheless, collaborators at traditional tables regularly reach for task artefacts, suggesting a reasonable balance exists. Morris et al. [12] investigated reach on tabletop interfaces in a study comparing a single central shared menu versus replicated menus near to each collaborator. Participants were not concerned by the ergonomics of reaching the shared central menu. Participants were, however, concerned by the sociallyawkward physical proximity to others' hands when using the shared menu, although this is perhaps because the task required frequent menu use by different collaborators. Such physical proximity does not occur in remote tabletops and is in any case unlikely for infrequently-used actions such as moving the floor plan.

\section{Limitations and Future Work}

Further work is required to establish the effects of remote tabletop collaboration for tasks in which a lack of spatial partitioning is particularly disadvantageous, and the effectiveness of interventions such as the proposed visual boundaries and coloured palettes. It is also unclear how the findings extend to larger groups in which some 
collaborators are co-located around a table, or to larger groups of mutually remote collaborators.

Further work is also required to evaluate the proposed interaction techniques themselves (such as dragging from a point to create a menu). Future work might also consider how to apply the properties of localised and continuous incremental visual changes to other remote collaboration interfaces. Scrolling in conventional GUI groupware, for instance, is detrimental to workspace awareness [5]. Alternative techniques (such as task artefacts that can be moved, shrunk and piled, as is commonplace in tabletop interfaces) might therefore be applied to avoid scrolling in conventional groupware and so address this issue. Finally, novel spatial video and 3-D projection technologies might address the lack of 3-D depth cues in remote arm representations and hence the problem of leaning to gesture, in remote tabletops and other large-format interfaces.

\section{CONCLUSIONS}

We have presented an exploratory study of two work practices in remote tabletop interfaces. We investigated colocated tabletop collaboration and also remote tabletop collaboration using two remote seating arrangements. Remote tabletop interfaces did not afford territorial partitioning of space in the way observed in co-located collaboration, in either of the remote seating arrangements. We suggest the effects of reaching caused this difference. Both remote and co-located tabletop interfaces afforded individual and group work as part of a shared task. However, two interaction techniques impaired workspace awareness, and so the ability to transition fluidly between coupling styles. We suggest a lack of localised interaction, and a lack of continuous incremental visual changes, caused these problems. The findings yield implications for the design and further study of remote tabletops.

\section{ACKNOWLEDGEMENTS}

We gratefully acknowledge Alan Blackwell, Darren Edge, Cecily Morrison, and the anonymous referees, for feedback on drafts; and the EPSRC and Thales Research and Technology (UK), who funded this work.

\section{REFERENCES}

1. Ashdown, M. and Robinson, P. Escritoire: A Personal Projected Display. IEEE Multimedia, 12, 1 (2005), 34-42.

2. Bier, E. A., Stone, M., Pier, K., Buxton, W., and DeRose. T. Toolglass and Magic Lenses: the See-Through Interface. Proc. SIGGRAPH 1993, 73-80.

3. Dourish, P. and Bellotti, V. Awareness and Coordination in Shared Workspaces. Proc. CSCW 1992, 107-114.

4. Gutwin, C. and Greenberg, S. Design for Individuals, Design for Groups: Tradeoffs between Power and Workspace Awareness. Proc. CSCW 1998, 207-216.

5. Gutwin, C. and Greenberg, S. A Descriptive Framework of Workspace Awareness for Real-Time Groupware. Comput. Supported Coop. Work 11, 3 (Nov. 2002), 411-446.
6. Ha, V., Inkpen, K. M., Mandryk, R. L., Whalen, T. Direct Intentions: The Effects of Input Devices on Collaboration around a Tabletop Display. Proc. TABLETOP 2006, 177-184.

7. Hall, E. T. Distances in Man: The Hidden Dimension. Double Day, Garden City, NY, 1966.

8. Hutterer, P., Close, B. S., and Thomas, B. H. Supporting Mixed Presence Groupware in Tabletop Applications. Proc. TABLETOP 2006, 63-70.

9. Izadi, S., and Agarwal, A. C-Slate: Exploring Remote Collaboration on Horizontal Multi-touch Surfaces. Proc. TABLETOP 2007, 3-10.

10. Kirk, D. Turn It This Way: Remote gesturing in VideoMediated Communication. Ph.D. Thesis, University of Nottingham, 2007.

11. Kruger, R., Carpendale, S., Scott, S.D., and Tang, A. Fluid Integration of Rotation and Translation. Proc. CHI 2005, 601610 .

12. Morris, M. R., Paepcke, A., Winograd, T., and Stamberger, J. Teamtag: Exploring Centralized versus Replicated Controls for Co-Located Tabletop Groupware. Proc. CHI 2006, 12731282.

13. Nacenta, M. A., Pinelle, D., Stuckel, D., and Gutwin, C. The Effects of Interaction Technique on Coordination in Tabletop Groupware. Proc. Graphics Interface 2007, 191-198.

14. Pauchet, A., Coldefy, F., Lefebvre, L., Louis dit Picard, S., Perron, L., Guérin, J. Tabletops: Worthwhile Experience of Collocated and Remote Collaboration. Proc. TABLETOP 2007, 27-34.

15. Rogers, Y., and Lindley, S. Collaborating Around Vertical and Horizontal Large Interactive Displays: Which Way is Best? Interacting with Computers 16, 6 (2004), 1133-1152.

16. Scott, S. D., Carpendale, M.S.T,, and Habelski, S. 2005. Storage Bins: Mobile Storage for Collaborative Tabletop Displays. IEEE Comput. Graph. Appl. 25, 4 (Jul. 2005), 58-65.

17. Scott, S.D., Carpendale, M.S.T., and Inkpen, K.M. Territoriality in Collaborative Tabletop Workspaces. Proc. CSCW 2004, 294-303.

18. Tang, A., Neustaedter, C. and Greenberg, S. VideoArms: Embodiments for Mixed Presence Groupware. Proc. HCI 2006, 85-102.

19. Tang, A., Tory, M., Po, B., Neumann, P., and Carpendale, S. Collaborative Coupling Over Tabletop Displays. Proc. CHI 2006, 1181-1190.

20. Tang, J. C. Findings from Observational Studies of Collaborative work. Int. J. Man-Mach. Stud. 34, 2 (Feb. 1991), 143-160.

21. Tang, J. C. and Minneman, S. VideoWhiteboard: Video Shadows to Support Remote Collaboration. Proc. CHI 1991, 315-322.

22. Tse, E., Shen, C., Greenberg, S., and Forlines, C. Enabling Interaction with Single User Applications through Speech and Gestures on a Multi-User Tabletop. Proc. AVI 2006, 336-343.

23. Tuddenham, P., and Robinson, P. Distributed Tabletops: Supporting Remote and Mixed-Presence Tabletop Collaboration. Proc. TABLETOP 2007, 19-26.

24. Wellner, P. 1993. Interacting with Paper on the DigitalDesk. Commun. ACM 36, 7 (Jul. 1993), 87-96. 